\title{
The FIGMAS Online Database of Standards and Reference Materials - an Update
}

\author{
Emma Bullock ${ }^{1}$, William Nachlas ${ }^{2}$, Owen Neill ${ }^{3}$, Julien Allaz ${ }^{4}$ and Anette von der Handt ${ }^{5}$
}

${ }^{1}$ Carnegie Institution for Science, WASHINGTON, District of Columbia, United States, ${ }^{2}$ Department of Geoscience, University of Wisconsin-Madison, United States, ${ }^{3}$ University of Michigan, Ann Arbor, Michigan, United States, ${ }^{4}$ ETH Zürich, United States, ${ }^{5}$ Department of Earth and Environmental Sciences, University of Minnesota, Minneapolis, Minnesota, United States

Reference materials and standards (RMS) are essential when performing quantitative electron beam microanalysis. They are used for instrumental calibration, data quality assurance, comparison of results between different laboratories, and long-term checks on instrumental stability. A good RMS should be: compositionally homogeneous at the scale of analysis, chemically stable and resistant to degradation over time, easy to mount and polish, and readily available to all microbeam labs. In addition, RMS should have accurate, independently verified reference compositions. In some cases, crucial RMS have already or soon will become unavailable, so researchers continue to search for new materials to develop and test as RMS [1-3]. Unfortunately, practical constraints often make the development and formal certification of RMS difficult [4, 5], and leave analysts with a set of secondary or working measurement standards instead of certified standards. Such reference materials may suffer from shortcomings such as questionable provenance, homogeneity or impurity issues, contamination, or incomplete or inaccurate reference compositions. Furthermore, variations between individual batches and/or updated reference compositions [1,6] can lead to confusion and the potential risk of mixing old and new values.

To address these concerns, the Focused Interest Group on Microanalytical Standards (FIGMAS) was founded in 2016 as a joint project between the Microanalytical Society (MAS) and the Microscopy Society of America (MSA). FIGMAS aims to catalog existing RMS currently used for quantitative microanalysis, facilitate the development of new RMS, and create a centralized location to display information about RMS available to the analytical community. To this end, FIGMAS has created an online database [7] of existing RMS and is working with academic, federal, and commercial partners to identify, characterize, and manufacture a suite of new RMS materials to meet the needs of the modern quantitative microanalytical capabilities.

The database is a work in progress, and new entries are continuously added to it, with an emphasis on materials which are widely available for community use. FIGMAS members can edit the database, and are strongly encouraged to create entries both for RMS not currently listed in the database and for listed RMS for which alternate compositions exist depending on source, lot, and/or reference. Each RMS in the database contains information on the chemical composition of the material and relevant metadata such as representative EDS spectra, citations for the source of the reference values, and information on homogeneity and impurities. We are also working to identify and highlight reliable sources from which to obtain material, as availability is a crucial aspect of an RMS for most laboratories.

In order to evaluate new materials that may become potential RMS, FIGMAS has initiated a "Round Robin" of materials that are potentially chemically homogeneous at the micrometer scale and from grain to grain, and abundant enough to be widely available for the foreseeable future to all labs that request such a material. The Round Robin's first stage has been to identify a suite of silicate, phosphate and carbonate minerals and glasses from reputable sources. These have been mounted up (figure 1), polished, coated, and are currently in the process of being characterized by a suite of labs to a) test the suitability of the material as a reference standard and b) to check for any systematic issues with individual laboratory analyses. The results will be compiled and made available to the community, and preliminary results will be presented at the meeting. 
The goal of FIGMAS is to facilitate the use and commutability of standards and reference materials across the microanalytical community. We have created a database to widely and systematically disseminate information about RMS commonly used for X-ray microanalysis. We hope that this effort will help to move towards consensus on evaluating some of the materials used as RMS and to direct new microanalysts to welldocumented resources to develop and expand laboratory standard collections. To prosper, the database requires contributions from FIGMAS members to augment gaps in the existing collection. The authors thank members of the community who generously donate their time and information, and hope that new analysts will continue to contribute to this effort in the future.

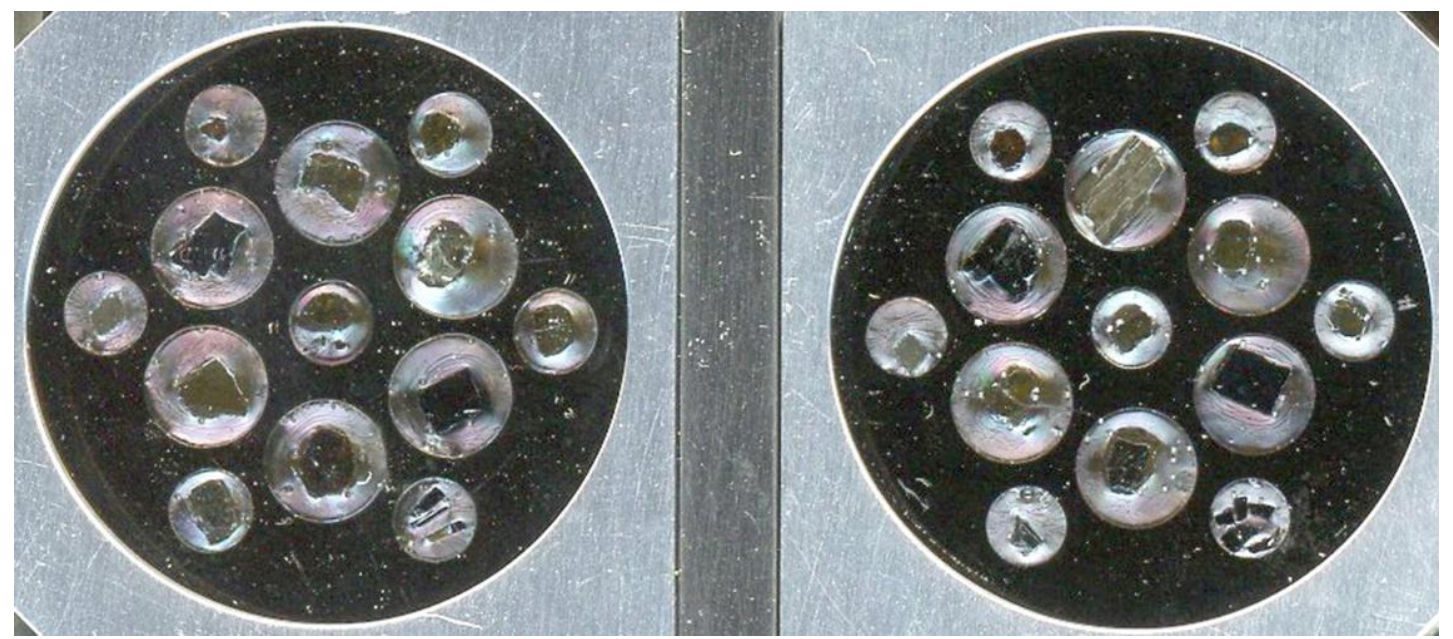

Figure 1. Two of the five 1" polished mounts prepared for the Round Robin. Each mount contains an identical suite of silicate, carbonate, and phosphate minerals and glasses.

\section{References}

[1] Donovan J, et al. 2003 Can. Mineralogist 41 221-232

[2] Carpenter P K, et al. 2002 J. Res. Nat. Inst. Stand. Tech. 107 703-718

[3] Vicenzi E P, et al. 2002 J. Res. Nat. Inst. Stand. Tech. 107 719-727

[4] Marinenko RB 2002 J. Res. Nat. Inst. Stand. Tech. 107 687-691

[5] May W, et al. NIST Special Publication 260-136

[6] Wagner B, et al. 2012 Anal. Bioanal. Chem. 402 1667-1677

[7] "FIGMAS - Home" https://figmas.org (accessed Feb 15, 2021) 